\title{
Fibromyalgia syndrome in chronic hemodialysis patients is associated with depression, hypoalbuminemia and inflammation
}

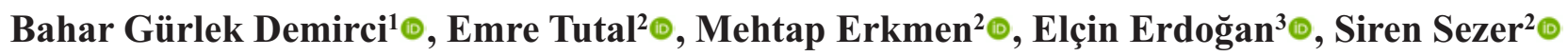 \\ ${ }^{1}$ Department of Nephrology, Ylldırım Beyazıt University School of Medicine, Ankara, Turkey \\ ${ }^{2}$ Department of Nephrology, Başkent University School of Medicine, Ankara, Turkey \\ ${ }^{3}$ Department of Internal Medicine, Başkent University School of Medicine, Ankara, Turkey
}

DOI: $10.18621 /$ eurj.423605

\begin{abstract}
Objectives: Fibromyalgia syndrome (FMS) is an entity that presents with wide-spread chronic pain in musculoskeletal system, disturbed sleep, reduced mental functioning and depressed mood. We aimed to determine the incidence of FMS in our hemodialysis patients and to evaluate the association between FMS, depression and inflammation in patients ongoing hemodialysis.

Methods: Among 169 patients, 140 patients were enrolled into our study. Twenty-nine patients were excluded according to exclusion criteria. Demographic characteristics including age, sex, body mass index, duration of dialysis, the etiology of end stage renal disease, the dialysis adequacy $(\mathrm{Kt} / \mathrm{V})$ were also recorded. All patients were evaluated through 2010 ACR preliminary diagnostic criteria including widespread pain index (WPI) and total symptom severity. All subjects completed Beck depression inventory (BDI) to determine psychological status.

Results: Among 140 hemodialysis patients, 76 (54.2\%) patients presented chronic widespread pain and 20 patients (14.2\%) met the 2010 ACR criteria for FMS. Patients were divided into FMS $(n=20 ; 14.2 \%)$ and non-FMS $(\mathrm{n}=120 ; 85.8 \%)$ groups. FMS group had significantly higher serum C-reactive protein levels and lower serum albumin levels when compared to non-FMS group. BDI $(31.4 \pm 1.4$ vs $14.8 \pm 0.6, p<0.005)$, WPI $(11.2 \pm 0.7$ vs $2.8 \pm 0.2, p=0.002)$ and symptom severity $(7.3 \pm 0.3$ vs $3.7 \pm 0.1, p<0.005)$ scores were significantly higher in FMS group.

Conclusions: The FMS itself and the related symptoms were correlated with depression in maintenance hemodialysis patients. Besides, inflammation and perhaps malnutrition-inflammation sydrome may trigger FMS in this population.
\end{abstract}

Keywords: Fibromyalgia, hemodialysis, depression

Received: May 15, 2018; Accepted: July 12, 2018; Published Online: August 5, 2018

$\mathbf{F}$ ibromyalgia is an idiopathic, chronic, nonarticular pain syndrome with generalized tender points. It is a multisystem disease characterized by sleep disturbance, fatigue, headache, morning stiffness, paresthesias, and anxiety. Fibromyalgia syn- drome (FMS) is an entity that presents with widespread chronic pain in musculoskeletal system, disturbed sleep, reduced mental functioning and depressed mood $[1,2]$. The prevalence of FMS in the general population has been estimated between $2.9 \%$ -

Address for correspondence: Bahar Gürlek Demirci, MD., Ylldırım Beyazıt University School of Medicine, , Department of Nephrology, 06800 Bilkent, Ankara, Turkey

E-mail: bahargurlek@gmail.com,Tel:+90 3122912525 
$5 \%$ [3]. Up to now, the classification criteria suggested by the American College of Rheumatology (ACR) in 1990 had been used in the diagnosis of fibromyalgia. According to this classification, there has to be left, right, top, bottom of body and axial skeletal chronic whole body pain for at least 3 months, and 11 or more tender points from the pre-defined 18 tender points for patient to be diagnosed as fibromyalgia [1]. However there are also some cases where 11 or more tender point criteria is not fulfilled so there are limitations in objectivity and usefulness of this diagnostic criteria [4]. Because of the kind of patient existing classification criteria, ACR suggested a new classification criteria in 2010 that diagnoses fibromyalgia as the sum of widespread pain index (WPI) and total symptom severity being more than a certain score, continuing symptoms for more than 3 months, and all three criteria without disease related to symptoms being satisfied [5]. The WPI is a measure of the number of painful body legions from a defined list of 19 areas. The symptom severity score includes an estimate of the degree of fatigue, waking unrefreshed, and cognitive symptoms and a number of somatic symptoms in general. The symptom severity scale may also be used for the assessment of patients with current and previous fibromyalgia or for longitudinal evaluation.

FMS is relatively frequent in the general population and affects females more often than males. Its prevalence is reported to increase with age [6]. Although FMS etiology and pathophysiology are still unclear, the current hypotheses focus on the central mechanisms of pain modulation and amplification in the genesis of FMS. Because musculoskeletal pain is regarded as the most prevalent form of chronic pain in end stage renal disease, a differential diagnosis of FMS should be considered in that group of patients. It may be difficult to determine if symptoms are related to FMS or the associated illness. Inflammatory rheumatic diseases, non-inflammatory musculoskeletal disorders, functional somatic syndromes, localized pain syndromes and anxiety disorders may coexist with FMS in end stage renal disease patients.

In our study, we aimed to determine the incidence of FMS in our hemodialysis patients and to evaluate the association between FMS, depression and inflammation in patients ongoing maintenance hemodialysis.

\section{METHODS}

Among 169 patients (male 63.3\%) with end stage renal disease who received 4 hours 3 sessions/week, maintenance hemodialysis in Başkent University Hospital hemodialysis centre, 140 patients were enrolled into our study. Patients having unregulated diabetic disease, thyroid disorders, chronic rheumatic diseases and neuropathic diseases, co-existing liver disease, solid organ or hematological malignancy, immobilization, overt cardiovascular disease were excluded from the study. Twenty-nine patients were excluded according to exclusion criteria. We have received a consent form from all the patients. Demographic characteristics including age, sex, body mass index (BMI; $\mathrm{kg} / \mathrm{m}^{2}$ ), duration of dialysis, the etiology of end stage renal disease, the dialysis adequacy $(\mathrm{Kt} / \mathrm{V})$ were recorded. All patients were evaluated through 2010 ACR preliminary diagnostic criteria including WPI and symptom severity scale [5]. Subjects were asked if they had experienced chronic widespread pain for at least 3 months. Each subject was also examined for tenderness at 18 tender points by digital palpation. One score point was assigned for each tender point noted; thus, each individual's tender point score was between 0 and 18 .

Patients were divided into FMS $(\mathrm{n}=20 ; 14.2 \%)$ and non-FMS) $(\mathrm{n}=120 ; 85.8 \%)$ groups. All subjects in FMS and non-FMS groups completed Beck depression inventory (BDI) to determine psychological status [7]. The BDI is a 21-question multi-choice self-report inventory that is one of the most widely used instruments for measuring the severity of depression. It is composed of items relating to depression symptoms such as hopelessness and irritability, cognitions such as guilt or feelings of being punished, as well as physical symptoms such as fatigue, weight loss, and lack of interest in sex. It measures depression through scores, which can range from 0 to 63 . The higher score is associated with the higher severity of depression. At the diagnosis of FMS in the current study, no patients were using specific medications for FMS, such as opioid analgesics or anti-depressants. As part of our quality assurance programme, all maintenance hemodialysis patients complete blood count and biochemical parameters were checked during monthly clinic visit within this 
period. In all participants, a venous blood sample was collected after an overnight fast to measure the concentration of the following biochemical variables using standard laboratory techniques: calcium, phosphorus, albumin, C-reactive protein (CRP), parathyroid hormone (PTH) levels. (iPTH; by chemiluminescence immunoassay $\left(\right.$ Cobast $^{\circledR}$, Roche Diagnostics $\mathrm{GmbH}$ ) levels. Body weight and BMI were taken after dialysis session. Height was obtained from the patient's chart.

\section{Statistical Analysis}

The Statistical Package for the Social Sciences (SPSS, version 15.0, SPSS Inc., Chicago, IL) was used to process and statistical analyses of all data. The results of monthly laboratory tests were reviewed and mean values of the tests were used for statistical correlation analyses. Data are presented as mean $\pm \mathrm{SD}$ and as percentages when expressing frequency. The comparison between FMS and non-FMS patients were evaluated by student's $t$ test for independent samples. Relationships between FMS and non-FMS or any parameters from the questionnaires were evaluated by Fisher's exact test. Pearson and Spearman rank tests were used to assess correlations between measurable variables, according to their parametric distribution, respectively. A $p$ value $<0.05$ was considered statistically significant.

\section{RESULTS}

Among 140 hemodialysis patients, 76 (44.9\%) patients presented chronic widespread pain and 20 patients (14.2\%) met the 2010 ACR criteria for FMS. Patients were divided into FMS $(\mathrm{n}=20 ; 14.2 \%)$ and non-FMS $(n=120 ; 85.8 \%)$ groups. Mean age of FMS and non-FMS groups were $55.2 \pm 8.3$ years and $54.5 \pm$ 13.9 years, respectively $(p=0.520$. No difference was obtained between FMS and NFMS patients according to their age, dialysis adequacy, duration of dialysis or marital status. In FMS and non-FMS groups, mean serum calcium levels were $9.1 \pm 0.5 \mathrm{mg} / \mathrm{dL}$ and $9.0 \pm$ $0.6 \mathrm{mg} / \mathrm{dL}$, respectively $(p=0.649)$; mean serum phosphorus levels were $4.9 \pm 0.9 \mathrm{mg} / \mathrm{dL}$ and $4.9 \pm 1$ $\mathrm{mg} / \mathrm{dL}$, respectively $(p=0.832)$; mean serum PTH levels were $439.5 \pm 32.4 \mathrm{mg} / \mathrm{dL}$ and $481.3 \pm 46.5$ $\mathrm{pg} / \mathrm{dL}$, respectively $(p=0.180)$. FMS group had

Table 1. The clinical and biochemical findings of study population

\begin{tabular}{lccc}
\hline & $\begin{array}{c}\text { FMS Group } \\
(\mathbf{n = 2 0 )}\end{array}$ & $\begin{array}{c}\text { Non-FMS Group } \\
(\mathbf{n = 1 2 0})\end{array}$ & p value \\
\hline Age (years) & $55.2 \pm 8.3$ & $54.5 \pm 13.9$ & 0.520 \\
Male gender, n (\%) & $12(60)$ & $78(65)$ & 0.623 \\
Chronic kidney disease etiology & & & 0.814 \\
Diabetes Mellitus, n (\%) & $10(50.0)$ & $61(50.8)$ & \\
Hypertension, n (\%) & $6(30.0)$ & $14(11.6)$ & \\
Glomerulonephritis, n (\%) & $2(10.0)$ & $6(5.0)$ & \\
Polycyctic kidney disease, n (\%) & $1(5.0)$ & $2(1.6)$ & \\
Others, n (\%) & $1(5.0)$ & $4(3.3)$ & 0.516 \\
Duration of dialysis (years) & $9.3 \pm 0.8$ & $10.7 \pm 0.4$ & 0.514 \\
Fasting glucose (mg/dL) & $96.4 \pm 2,6$ & $98.6 \pm 1.8$ & 0.649 \\
Calcium (mg/dL) & $9.1 \pm 0.5$ & $9.0 \pm 0.6$ & 0.832 \\
Phosphorus (mg/dL) & $4.9 \pm 0.9$ & $4.9 \pm 1.0$ & 0.180 \\
PTH ( pg/mL) & $493.5 \pm 32.4$ & $481.0 \pm 46.5$ & $\mathbf{0 . 0 0 1}$ \\
Albumin (g/dL) & $3.1 \pm 0.2$ & $3.9 \pm 0.3$ & $\mathbf{0 . 0 0 1}$ \\
CRP (mg/L) & $13.8 \pm 15.1$ & $8.1 \pm 13$ & 0.418 \\
Hemoglobin (g/dL) & $10.6 \pm 1.2$ & $11.3 \pm 1.2$ & 0.316 \\
BMI (kg/m $\left.{ }^{2}\right)$ & $21.4 \pm 1.7$ & $24.2 \pm 2.1$ & $\mathbf{0 . 0 0 2}$ \\
WPI & $11.2 \pm 0.7$ & $2.8 \pm 0.2$ & $\mathbf{0 . 0 0 5}$ \\
SS & $7.3 \pm 0.3$ & $3.7 \pm 0.1$ & $\mathbf{0 . 0 0 2}$ \\
BDI & $34.1 \pm 1.4$ & $14.8 \pm 0.6$ & \\
\hline Data are shown as & &
\end{tabular}

Data are shown as mean \pm standard deviation or number $(\%)$. BDI $=$ Beck depression inventory, $\mathrm{BMI}=$ body mass index, $\mathrm{CRP}=\mathrm{C}$-recative protein, $\mathrm{FMS}=$ fibromyalgia, $\mathrm{PTH}=$ parathyroid hormone, $\mathrm{SS}=$ symptom severity, WPI = widespread pain index 
significantly higher serum CRP $(13.8 \pm 15.1$ vs $8.1 \pm$ $13 \mathrm{mg} / \mathrm{L}, p=0.01$ ) whereas lower serum albumin levels $(3.1 \pm 0.2 \mathrm{~g} / \mathrm{L}$ and $3.9 \pm 0.3 \mathrm{~g} / \mathrm{L}, \mathrm{p}=0.001)$ when compared to non-FMS group. Hemoglobin levels were similar between FMS and non-FMS groups $(10.6 \pm 1.2 \mathrm{~g} / \mathrm{dL}$ and $11.3 \pm 1.2 \mathrm{~g} / \mathrm{dL}$, respectively). $\mathrm{BDI}(31.4 \pm 1.4$ vs $14.8 \pm 0.6, p<$ $0.005)$, WPI $(11.2 \pm 0.7$ vs $2.8 \pm 0.2, p=0.002)$ and symptom severity $(7.3 \pm 0.3$ vs $3.7 \pm 0.1, p<0.005)$ scores were significantly higher in FMS group (Table $1)$.

\section{DISCUSSION}

To our knowledge, present study is the first in the literature regarding clinical and laboratory outcomes of fibromyalgia in addition to impact on depression in maintenance hemodialysis patients. Fibromyalgia is usually considered as a disorder of females 20 to 50 years of age; however, it also has been observed in males, children, adolescents, and older persons [8,9]. A study by Wolfe et al. [6] with screening of 3006 adults revealed FMS prevalence rates as $3.4 \%$ in female and $0.5 \%$ in male. The recent study in the literature about fibromyalgia in maintenance hemodialysis patients reported that the prevalence rates in the maintenance hemodialysis and control groups were statistically similar to work by Wolf et al. [6]. In accord with the literature, present study had similar prevalence rates in hemodialysis and control groups; however the overall rate of fibromyalgia was higher when compared to other studies. We can explain this higher prevalence with the 2010 preliminary classification criteria according to American College of Rheumatology (ACR) that we used for the FMS diagnosis, whereas the recent studies were diagnosing FMS with ACR classification criteria of 1990. Yuceturk et. al. [10] reported that FMS was more frequent in females, with rates of $10.4 \%$ in women and $1.9 \%$ in men. In contrast to the most studies FMS was more common in men, in current study. This conflicting result was not surprising because the percentage of men in the study was $63 \%$. Fibromyalgia is a neurogenic inflammatory response to allergens, infectious agents, chemicals or emotional stress; however the sources of inflammation triggering the fibromyaigia syndrome remain to be unknown
[11]. In our study, mean serum CRP levels were significantly higher in fibromyagia group, as expected. Although we didn't evaluate other inflammation markers as TNF- $\alpha$, IL- 6 and IL- 8 as a limitation of this study. High levels of serum CRP reflects the importance to take into account the inflammation in patients with fibromyalgia to project the therapeutic approach. The index for dialysis adequacy $(\mathrm{Kt} / \mathrm{V})$ and nutritional status are known to be determinants for health status in the hemodialysis patients and affect their quality of life (QOL). In present study, while there was no significant difference for dialysis adequacy between two groups, hypoalbuminemia that is a major marker for malnutrition and a well-known negative acute phase reactant was significantly lower in FMS patients when compared to non-FMS patients in our study. This finding may be a result of malnutrition-inflammation syndrome that can trigger FMS in maintenance hemodialysis patients. We also couldn't detect an association between FMS and serum levels of PTH, alkaline phosphatase, calcium or phosphorus. Although this result was similar to the literature, we wonder for we anticipate high levels of parathormone and phosphorus levels that were wellknown musculoskeletal pain causes in hemodialysis patients.

Chronic pain has been reported as a leading cause of insomnia in any medical illness where most studies have focused on rheumatic disorders for these symptoms [12]. Sleep difficulties and related symptoms as fatigue, paraesthesia and morning stiffness have been reported in $>75 \%$ of patients with fibromyalgia [13]. In Yuceturk et al.'s study [10], the prevalence of fatigue, sleep disturbances and restless leg syndrome were higher in maintenance hemodialysis patients than in control subjects, regardless of whether or not the individual was diagnosed as having FMS. However all these entities were more common but not statistically significant in maintenance hemodialysis patients with proven FMS in current study.

Finally, we focused on depression which characteristically presents in FMS patients. The percentage of depressive symptoms is high in patients with FMS, ranging from $40 \%$ to $80 \%[14,15]$.

Thus, similar to previous studies we showed the negative impact of fibromyalgia on quality of life by chronic pain and related symptoms, we observed 
significantly higher BDI, WPI and symptom severity scores in FMS patients, as expected and then referred patients to psychiatry department. In conclusion, although several studies have evaluated the coexistence of FMS and psychiatric disorders, data about the fibromyalgia and depression in hemodialysis patients is limited and need further studies with higher number of subjects.

\section{Limitations}

There were several limitations of current study: 1)The study especially FMS group have small sample size. 2)- We assess the relation between FMS and inflammation only with serum CRP and albumin levels, we may invigorate with evaluating other inflammation markers as IL-6, IL-8 and TNF-alpha. 3)- We didn't evaluate the questionnaires for assessing the quality of life and sleep disturbance.

\section{CONCLUSION}

In present study the prevalence of FMS in hemodialysis patients was $11.8 \%$. The FMS itself and the related symptoms were correlated with depression. Moreover, inflammation and perhaps malnutritioninflammation syndrome may trigger FMS. Thus patients with FMS and ongoing maintenance hemodialysis should be followed closely for inflammation and mood disorders.

\section{Conflict of interest}

The authors disclosed no conflict of interest during the preparation or publication of this manuscript.

\section{Financing}

The authors disclosed that they did not receive any grant during conduction or writing of this study.

\section{REFERENCES}

[1] Wolfe F, Smythe HA, Yunus MB, Bennett RM, Bombardier $\mathrm{C}$, Goldenberg DL, et al. The American College of
Rheumatology 1990 Criteria for the Classification of Fibromyalgia. Report of the Multicenter Criteria Committee. Arthritis Rheum 1990;33:160-72.

[2] Bernardy K, Klose P, Welsch P, Häuser W. Efficacy, acceptability and safety of internet-delivered psychological therapies for fibromyalgia syndrome - A systematic review and meta-analysis of randomized controlled trials. Eur J Pain 2018 Jul 8. doi: 10.1002/ejp.1284.

[3] Biewer W, Conrad I, Häuser W. [Fibromyalgia]. Schmerz 2004;18:118-24. [Article in German]

[4] Fitzcharles MA, Boulos P. Inaccuracy in the diagnosis of fibromyalgia syndrome: analysis of referrals. Rheumatology (Oxford) 2003;42:263-7.

[5] Wolfe F, Clauw DJ, Fitzcharles MA, Goldenberg DL, Katz RS, Mease P, et al. The American College of Rheumatology preliminary diagnostic criteria for fibromyalgia and measurement of symptom severity. Arthritis Care Res (Hoboken) 2010;62:60010.

[6] Wolfe F, Ross K, Anderson J, Russell IJ, Hebert L. The prevalence and characteristics of fibromyalgia in the general population. Arthritis Rheum 1995;38:19-28.

[7] Julian LJ. Measures of anxiety: State-Trait Anxiety Inventory (STAI), Beck Anxiety Inventory (BAI), and Hospital Anxiety and Depression Scale-Anxiety (HADS-A). Arthritis Care Res (Hoboken) 2011;63 Suppl 11:S467-72.

[8] Chakrabarty S, Zoorob R. Fibromyalgia. Am Fam Physician 2007;76:247-54.

[9] Wernze H, Herdegen T. Long-term efficacy of spironolactone on pain, mood, and quality of life in women with fibromyalgia: An observational case series. Scand J Pain 2017;5:63-71.

[10] Yuceturk TE, Yucel AE, Yuceturk H, Kart-Koseoglu H, Unuvar R, Ozdemir FN, et al. Fibromyalgia: its prevalence in haemodialysis patients and its relationships with clinical and laboratory parameters. Nephrol Dial Transplant 2005;20:2485-8. [11] Omoigui S. The biochemical origin of pain: the origin of all pain is inflammation and the inflammatory response. Part 2 of 3 - inflammatory profile of pain syndromes. Med Hypotheses 2007;1169-78.

[12] Wolfe F . Fibromyalgia: the clinical syndrome. Rheum Dis Clin North Am 1989;15:1-18.

[13] Roizenblatt M, Rosa Neto NS, Tufik S, Roizenblatt S. Painrelated diseases and sleep disorders. Braz J Med Biol Res 2012;45:792-8.

[14] Homann D, Stefanello JM, Góes SM, Breda CA, Paiva Edos S, Leite N. Stress perception and depressive symptoms: functionality and impact on the quality of life of women with fibromyalgia. Rev Bras Reumatol 2012;52:319-30. [Article in English, Portuguese]

[15] Cavalcante AB, Sauer JF, Chalot SD, Assumpção A, Lage LV, Matsutani LA, et al. [The prevalence of fibromyalgia: a literature review]. Rev Bras Reumatol 2006;46:40-8. [Article in Portuguese] 